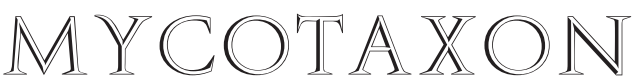

ISSN (print) 0093-4666 (online) 2154-8889 Mycotaxon, Ltd. ( $) 2017$

January-March 2017-Volume 132, pp. 107-123

http://dx.doi.org/10.5248/132.107

\title{
Cyanodermella asteris sp. nov. (Ostropales) from the inflorescence axis of Aster tataricus
}

Linda JAhN ${ }^{1,}$, Thomas SchafHaUser ${ }^{2}$, STEFAn PAN $^{2}$, Tilmann Weber ${ }^{2,7}$, Wolfgang Wohlleben ${ }^{2}$, David FeWer ${ }^{3}$, KaArina Sivonen ${ }^{3}$, Liane Flor ${ }^{4}$, Karl-Heinz van PÉE ${ }^{4}$, Thibault Caradec ${ }^{5}$, Philippe Jacques ${ }^{5,8}$, Mieke M.E. Huijbers ${ }^{6,9}$, Willem J.H. VAN BerKel ${ }^{6}$ \& JUTta LUdWig-MÜLleR ${ }^{1, *}$

${ }^{1}$ Institut für Botanik, Technische Universität Dresden, 01062 Dresden, Germany

${ }^{2}$ Mikrobiologie und Biotechnologie, Interfakultäres Institut für Mikrobiologie und Infektionsmedizin, Eberhard Karls Universität Tübingen, Auf der Morgenstelle 28, 72076 Tübingen, Germany

${ }^{3}$ Microbiology and Biotechnology Division, Dept. of Food and Environmental Sciences, University of Helsinki, Viikinkaari 9, FIN-00014, Helsinki, Finland

${ }^{4}$ Allgemeine Biochemie, Technische Universität Dresden, 01069 Dresden, Germany

${ }^{5}$ Laboratoire ProBioGEM, Université Lille1-Sciences et Technologies, Villeneuve d'Ascq, France

${ }^{6}$ Laboratory of Biochemistry, Wageningen University, Dreijenlaan 3, 6703 HA Wageningen, The Netherlands

${ }^{7}$ moved to: Novo Nordisk Foundation Center for Biosustainability, Technical University of Denmark, Kemitorvet Bygning 220, 2800 Kgs. Lyngby, Denmark

${ }^{8}$ moved to: Gembloux Agro-Bio Tech, Université de Liege, Passage des Déportés 2, 5030 Gembloux, Belgium

${ }^{9}$ moved to: Department of Biotechnology, Technical University Delft, Van der Maasweg 9, 2629 HZ Delft, The Netherlands

*CorReSPONDENCE To:Linda.Jahn@tu-dresden.de,Jutta.Ludwig-Mueller@tu-dresden.de

ABstract-An endophytic fungus isolated from the inflorescence axis of Aster tataricus is proposed as a new species. Phylogenetic analyses based on sequences from the ribosomal DNA cluster (the ITS1+5.8S+ITS2, 18S, and 28S regions) and the RPB2 gene revealed a relationship between the unknown fungus and the Stictidaceae lineage of the Ostropales. The new species, Cyanodermella asteris, grows in standard fungal growth media as a fluffy, pink filamentous fungus. Asexual and sexual sporulation has not yet been observed on media or in the plant.

KeY words-Ascomycota, Asteraceae, Lecanoromycetes, Pezizomycotina 
108 ... Jahn \& al.

\section{Introduction}

Aster tataricus (Asteraceae) is native to northern Asia in Siberia, Mongolia, Japan, Korea, and China. Its roots are well known in traditional Chinese and Japanese medicine due to their diuretic, antibacterial, antiviral, and anti-ulcer activities (Shao et al. 1997a, b; Shirota et al. 1997). Diverse secondary metabolites have been identified in A. tataricus roots: shiononetype triterpenes [e.g., astataricusones and astataricusol (Zhou et al. 2013); astershionones (Zhou et al. 2014)], cyclopeptides[e.g., astins (Morita et al. 1996, Xu et al. 2013)], and flavonoids [e.g., quercetin and kaempferol (Wang et al. 2003)].

With over 2700 species in eleven families, the Ostropales (Hibbett et al. 2007) represent a large part of the Lecanoromycetes (Ascomycota, Pezizomycotina). Although most ostropalean fungi are lichenized, parasites and saprotrophs are also known (Grube \& Hawksworth 2007; Lutzoni et al. 2004; Sherwood 1977a, 1977b; Wedin et al. 2004, 2006). Baloch et al. (2010), who outline the current taxonomy of the Ostropales, note that most Stictidaceae are saprotrophs and form very small fruiting bodies.

Here we describe our isolation of an endophytic fungus from the inflorescence of Aster tataricus and demonstrate its placement in the Ostropales. DNA sequences from the newly discovered fungus matched no previously sequenced species, although it did show phylogenetic affinities with Cyanodermella. For that reason, we propose this endophyte as a new species, named here Cyanodermella asteris.

\section{Materials \& methods}

Chemicals were purchased from Duchefa (Haarlem, The Netherlands) and Roth (Karlsruhe, Germany). Plant hormones were obtained from Duchefa and SigmaAldrich (Hamburg, Germany) and PCR reagents from Thermo Fisher Scientific (Waltham, USA).

\section{Collecting \& field sites}

The Aster tataricus host plant was obtained from Sarastro Stauden (Ort im Innkreis, Austria) and cultivated in the Plant Physiology laboratory greenhouse, Technische Universität Dresden, Germany. The cultivar is henceforth referred to as A. tataricus cv. Austria.

\section{Isolation}

The fungus was isolated from surface-sterilized inflorescence axes of Aster tataricus cv. Austria. After the axes were treated first for $30 \mathrm{~s}$ with $70 \%$ ethanol + $0.1 \%$ Triton $\mathrm{X}-100$, then for $5-7$ min with $4.2 \%$ sodium hypochlorite $+0.1 \%$ Triton $\mathrm{X}-100$, and washed 3 times with autoclaved distilled water, samples were cultivated 


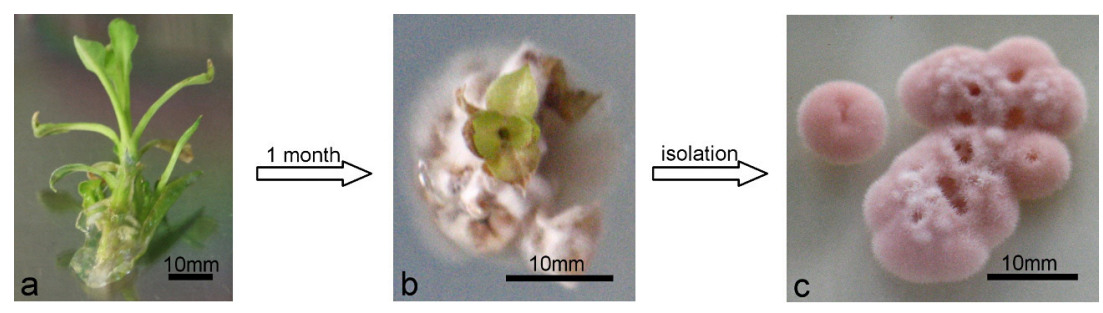

FIG. 1. Isolation of the new endophyte Cyanodermella asteris. a. During the first few weeks, the new endophyte $C$. asteris was isolated from sterile plantlets growing on hormone containing MS medium. b. Antimycotic treatment did not reduce the fungal outgrowth, and the fungus still appeared on the plant surface and grew on the MS medium. c. Isolation and cultivation on potato dextrose agar produced a fluffy pink filamentous fungus.

on Murashige-Skoog (MS) medium (Murashige-Skoog 1962; 4.4 g/l MS medium including vitamins, $3 \%$ sucrose, $1 \%$ phyto agar, $\mathrm{pH} 5.8$ ) including auxins and/or cytokinins (i.e., naphtylacetic acid, indole-3-butyric acid, 6-benzylaminopurine) usually used to stimulate sterile plants in vitro. The MS plates were incubated in long day cycles $\left(16 \mathrm{~h}\right.$ light, $23^{\circ} \mathrm{C} ; 8 \mathrm{~h}$ dark, $\left.18^{\circ} \mathrm{C}\right)$. After several transfers of sterile plantlets to fresh MS plates, we found a fungus growing from the small plants into the MS medium. Initially considered a contaminant, the fungus was treated with antimycotics $\left(50 \mu \mathrm{g} / \mathrm{ml} \mathrm{Nystatin}{ }^{\oplus}\right.$ for 4 months and penicillin/ streptomycin/ amphotericin B solution for 1 month). However, the treatment did not eliminate the fungus from the plant culture. In order to identify the fungus, we isolated hyphae from the plantlets for cultivation on potato dextrose $(26.5 \mathrm{~g} / \mathrm{l}$ potato dextrose bouillon, $1 \%$ phyto agar, $\mathrm{pH} 5.8$ ) and malt extract agar (3\% malt extract, $1 \%$ phyto agar, $\mathrm{pH} 5.8$ ) in an incubator in the dark at $28^{\circ} \mathrm{C}$ (FIG.1).

\section{DNA extraction, PCR amplification, and sequencing}

DNA was extracted from mycelium grown in potato dextrose broth following a modified protocol by Möller et al (1992) and precipitated with isopropanol. The mixture was left overnight at $-20^{\circ} \mathrm{C}$, spun down, and the DNA pellet washed three times with $70 \%$ ethanol to ensure salt-free DNA. The DNA was amplified using Phusion ${ }^{\star}$ polymerase according to the LifeTechnologies instruction manual. The primers $(0.5 \mu \mathrm{M})$ used for both PCR and sequencing were ITS1F \& ITS4 and NS3 \& NS8 (White et al. 1990), LROR \& LR7 (Vilgalys \& Hester 1990), and FRPB2-5F, $-7 \mathrm{CR},-7 \mathrm{CF}, \&-11 \mathrm{AR}$ (Liu et al. 1999). PCR conditions were initial denaturation at $98^{\circ} \mathrm{C}$ for $30 \mathrm{~s}, 35$ cycles of $98^{\circ} \mathrm{C}$ for $10 \mathrm{~s}$, appropriate annealing temperature for $30 \mathrm{~s}$, and $72^{\circ} \mathrm{C}$ for $1 \mathrm{~min}$ followed by a final elongation at $72^{\circ} \mathrm{C}$ for $5 \mathrm{~min}$. Amplification was carried out in an Eppendorf Mastercycler ep gradient S. PCR products were separated on a 1xTAE gel, cut out, extracted using a GE Healthcare Gel Extraction Kit, and sequenced at Eurofins MWG (Ebersberg, Germany). 
Total DNA was isolated from plants as described above and checked with the appropriate fungal primers under the same PCR conditions except for an annealing temperature of $68^{\circ} \mathrm{C}$. The PCR fragments were also sequenced and compared with the known rDNA sequence of the endophytic fungus to confirm the presence of the fungus in the plants.

\section{Phylogenetic analysis}

Sequences of the nuclear internal transcribed spacer region (ITS, including ITS1+5.8S rDNA+ITS2), the large (28S) and small (18S) nuclear rDNA subunits, and the second largest RNA polymerase II (RPB2) subunit from Cyanodermella asteris were run in a BLASTn search (Altschul et al. 1990). The different DNA regions were also used to build a phylogeny including closely related fungi. For this, the ITS, 18S, $28 \mathrm{~S}$ and RPB2 sequences from C. asteris and other fungi representing the Stictidaceae (TABLE 1) were aligned using the Guidance2 Server (Landan \& Graur 2008, Penn et al. 2010, Sela et al. 2015) with the default settings (including MAFFT algorithm) and Phlyctidaceae and Coenogoniaceae species as out-group. After sequence alignment, phylogenetic trees were generated from single region and combined sequences using MEGA6.0 (Tamura et al. 2013). The phylogenetic trees were inferred using the Maximum Likelihood method based on the General Time Reversible (Nei \& Kumar 2000) or the Kimura-2-parameter model (Kimura 1980). Initial tree(s) for the heuristic search were obtained by applying the Neighbor-Joining method to a matrix of pairwise distances estimated using the Maximum Composite Likelihood (MCL) approach. A discrete Gamma distribution was used to model evolutionary rate differences among sites. The bootstrap method was used with 1000 replicates (Felsenstein 1985).

The combined alignment and phylogenetic tree do not include all species, because rDNA cluster sequences were incomplete for some (TABLE 1).

\section{Microscopy}

The fungus was examined under an AP-8 binocular microscope (Thalheim Spezial Optik, Pulsnitz, Germany) and tissue samples were inspected with transmitted light with an Axiolab microscope (Zeiss, Jena, Germany). Additionally, fungal hyphae from a shake culture were stained with $1 \mathrm{~g}$ Congo Red in $100 \mathrm{ml}$ 5\% SDS to visualize fungal cell walls and septa (Clémençon 2009) under the microscope.

\section{Growth tests}

Malt extract broth $(50 \mathrm{ml})$ was inoculated with $0.5 \mathrm{ml}$ of homogenized hyphae of Cyanodermella asteris and incubated at $180 \mathrm{rpm}$. The medium was buffered in the $\mathrm{pH}$ test with $50 \mathrm{mM}$ phosphate or $50 \mathrm{mM}$ citrate buffer and cultures were incubated at $22^{\circ} \mathrm{C}$ for $10 \mathrm{~d}$. The cultures of the temperature test were incubated in unbuffered medium ( $\mathrm{pH} \mathrm{5.3)} \mathrm{for} 17 \mathrm{~d}$. Growth rates (mm/day) were recorded from $42 \mathrm{~d}$ old cultures on potato dextrose and malt extract agar plates kept at $23^{\circ} \mathrm{C}$ in the dark. Colors were coded according to Munsell (1905). 
TABLE 1. DNA sequences from Stictidaceae used for phylogenetic trees

\begin{tabular}{|c|c|c|c|c|c|}
\hline Species & $\begin{array}{l}{ }^{*}=\text { ISOLATE } \\
\wedge=\text { VOUCHER }\end{array}$ & $18 \mathrm{~S}$ & ITS & $28 \mathrm{~S}$ & $\mathrm{RPB}_{2}$ \\
\hline \multirow{2}{*}{$\begin{array}{l}\text { Absconditella } \\
\text { lignicola }\end{array}$} & ${ }^{\star} \mathrm{EB} 211$ & - & FJ904669.1 & FJ904669.1 & - \\
\hline & $\begin{array}{l}\text { Svensson \& Baloch } \\
\text { SW187 (S) }\end{array}$ & - & - & - & HM244776.1 \\
\hline A. rubra & $\wedge^{\wedge}$ van den Boom 52517 & KT454800.1 & KT 454800.1 & - & - \\
\hline \multirow[t]{3}{*}{ A. $s p$. } & ${ }^{\star}$ Spribille 39168 & KR017250.1 & KR017125.1 & KR017188.1 & KR017501.1 \\
\hline & $\wedge \mathrm{GS5} \_2 \_17$ & - & KF128882.1 & - & - \\
\hline & $\wedge$ Palice 3820 & - & - & $\mathrm{AY} 300825.1$ & - \\
\hline A. sphagnorum & ${ }^{\star} \mathrm{M} 24$ & EU940022.1 & JX298897.1 & EU940095.1 & EU940311.1 \\
\hline $\begin{array}{r}\text { Acarosporina } \\
\text { microspora }\end{array}$ & ${ }^{*}$ AFTOL 78 & AY 584667.1 & DQ782834.1 & AY 584643.1 & AY 584682.1 \\
\hline $\begin{array}{l}\text { Bryodiscus } \\
\quad \text { arctoalpinus }\end{array}$ & ^Baloch SW057 & - & - & нм244760.1 & HM244781.1 \\
\hline \multirow[t]{2}{*}{ Carestiella socia } & ${ }^{*} \mathrm{GG} 2410$ & - & AY 661687.1 & AY 661687.1 & - \\
\hline & $\wedge$ Wedin 7194 (UPS) & - & - & - & HM2 244782.1 \\
\hline $\begin{array}{l}\text { Coenogonium } \\
\text { leprieurii }\end{array}$ & $\begin{array}{l}\wedge \text { Kauff } \\
\quad \text { pa04021998-522 }\end{array}$ & AF465457.1 & - & AF 465442.1 & AY 641032.1 \\
\hline \multirow[t]{2}{*}{ C. luteum } & $\wedge$ Ryan 31430 (ASU) & AF279386.1 & - & AF279387.1 & AY 641038.1 \\
\hline & ${ }^{*}$ AFTOL 352 & - & HQ650710.1 & - & - \\
\hline \multirow[t]{2}{*}{ C. pineti } & ${ }^{\wedge} \mathrm{HB}$ Palice & - & - & AY 300834.1 & - \\
\hline & ^Thor 19164 (UPS) & - & - & - & HM244786.1 \\
\hline \multirow{3}{*}{$\begin{array}{l}\text { Conotrema } \\
\text { populorum }\end{array}$} & $\wedge \mathrm{UME} 41471$ & U 86582.1 & - & - & - \\
\hline & ${ }^{*} \mathrm{GG} 2610 \mathrm{a}$ & - & AY 527327.1 & - & - \\
\hline & $\wedge$ Gilenstam 2353 (UPS) & - & - & $\mathrm{AY} 300833.1$ & - \\
\hline \multirow[t]{2}{*}{ C. sp. } & ${ }^{*}$ MW7200 & & AY 527313.1 & AY 527313.1 & - \\
\hline & ${ }^{*} \mathrm{AN} 3222$ & - & AY 527336.1 & AY 527336.1 & - \\
\hline \multirow{3}{*}{$\begin{array}{l}\text { Cryptodiscus } \\
\text { foveolaris }\end{array}$} & ${ }^{\star} \mathrm{EB} 155$ & - & FJ904673.1 & - & - \\
\hline & ${ }^{\star} \mathrm{GG} 2603 \mathrm{a}$ & - & - & AY 661683.1 & - \\
\hline & $\begin{array}{l}\wedge \text { Baloch \& Arup } \\
\text { SW166 (S) }\end{array}$ & - & - & - & HM244787.1 \\
\hline \multirow[t]{2}{*}{ C. gloeocapsa } & ${ }^{*}$ EB93 & FJ904696.1 & FJ904674.1 & - & - \\
\hline & $\wedge$ Tibell 23543 (UPS) & - & - & - & HM2 24788.1 \\
\hline C. incolor & ${ }^{\star} \mathrm{S}: \mathrm{F} 116574$ & - & NR_121357 & - & - \\
\hline \multirow[t]{2}{*}{ C. pallidus } & *EB152 & - & FJ904679.1 & FJ904679.1 & - \\
\hline & $\begin{array}{l}\text { ^Baloch \& Wedin } \\
\text { SW174 (S) }\end{array}$ & - & - & - & HM244789.1 \\
\hline C. pini & $\begin{array}{l}\wedge \text { Baloch \& Arup } \\
\text { SW175 (S) }\end{array}$ & - & Hм 244762.1 & Hм 244762.1 & HM244790.1 \\
\hline C. rhopaloides & ${ }^{*} \mathrm{~EB} 100$ & - & FJ904685.1 & - & HM244791.1 \\
\hline C. sp.uncultured & clone F9 & - & KP323396.1 & - & - \\
\hline $\begin{array}{l}\text { Cyanodermella } \\
\text { asteris }\end{array}$ & $\begin{array}{l}\text { strain } \\
\quad \text { 03HOR06-2-4 }\end{array}$ & KT758843 & KT758843 & KT758843 & KU934214 \\
\hline
\end{tabular}


$112 \ldots$ Jahn \& al.

\begin{tabular}{|c|c|c|c|c|c|}
\hline Species & $\begin{array}{l}{ }^{*}=\text { ISOLATE } \\
\wedge=\text { VOUCHER }\end{array}$ & $18 \mathrm{~S}$ & ITS & $28 \mathrm{~S}$ & $\mathrm{RPB} 2$ \\
\hline C. oleoligni & strain DTO 301-G1 & KX999145.1 & KX950434.1 & KX950461.1 & KX999147.1 \\
\hline \multirow[t]{2}{*}{ C. viridula } & $\begin{array}{l}\text { ^E. \& C. Baloch } \\
\text { SW129 (S) }\end{array}$ & - & - & HM244763.1 & HM244792.1 \\
\hline & ^UME29146 & U86583.1 & - & - & - \\
\hline \multirow{3}{*}{$\begin{array}{l}\text { Glomerobolus } \\
\text { gelineus }\end{array}$} & *AFTOL 1349 & - & DQ247782.1 & - & DQ247793.1 \\
\hline & strain JK 5548K & DQ247811.1 & - & - & - \\
\hline & strain JK 5584C & - & - & DQ247798.1 & - \\
\hline $\begin{array}{l}\text { Odontotrema } \\
\text { phacidiellum }\end{array}$ & $\begin{array}{l}\wedge \text { Gilenstam } 2625 \\
\quad(\text { UPS) }\end{array}$ & - & - & HM244769.1 & HM 244802.1 \\
\hline O. phacidioides & $\wedge$ Palice $11440(\mathrm{~S})$ & - & - & HM244770.1 & Нм2 24803.1 \\
\hline \multirow[t]{2}{*}{ Ostropa barbara } & ${ }^{*}$ AFTOL 77 & AY 584666.1 & - & AY 584642.1 & - \\
\hline & $\begin{array}{l}\wedge \text { Wedin \& Baloch } \\
\text { SW071 (S) }\end{array}$ & - & HM244773.1 & - & HM244806.1 \\
\hline \multirow[t]{3}{*}{$\begin{array}{l}\text { Petractis } \\
\quad \text { luetkemuelleri }\end{array}$} & $\begin{array}{l}\text { ^Nimis \& Tretiach } \\
2000 \text { (TSB 31659) }\end{array}$ & AF465461.1 & - & AF465454.1 & - \\
\hline & *AFTOL 381 & - & HQ650714.1 & - & - \\
\hline & $\begin{array}{l}\text { ^Geletti \& Tretiach } \\
1995 \text { (TBS) }\end{array}$ & - & - & - & AY641061.1 \\
\hline \multirow[t]{2}{*}{ P. nodispora } & $\begin{array}{l}\text { ^A.Orange } 17559(\mathrm{NMW} \\
{[\text { C.2007.001.282]) }}\end{array}$ & FJ588712.1 & - & FJ588713.1 & - \\
\hline & ${ }^{\star} \mathrm{NMW}$ C.2007.001.284 & - & $N R \_120312.1$ & - & - \\
\hline \multirow[t]{2}{*}{ Phlyctis agelaea } & & - & - & HQ659177.1 & - \\
\hline & ^PHLAGE08257 & - & - & - & KCO2O280.1 \\
\hline \multirow[t]{2}{*}{ P. argena } & *AFTOL 1375 & DQ986725.1 & - & DQ986771.1 & DQ992458.1 \\
\hline & *BP8 & - & KJ409433.1 & - & - \\
\hline \multirow[t]{2}{*}{$\begin{array}{l}\text { Schizoxylon } \\
\text { albescens }\end{array}$} & $\begin{array}{l}\wedge \text { Gilenstam 2696a } \\
\quad(\text { UPS) }\end{array}$ & DQ401142.1 & NR_121319.1 & DQ401144.1 & - \\
\hline & ^Wedin 7919 (UPS) & - & - & - & HM244813.1 \\
\hline S. sp. & ${ }^{*} \mathrm{GG} 2365$ & - & AY661689.1 & AY661689.1 & - \\
\hline \multirow[t]{2}{*}{ Stictis brunnescens } & ${ }^{\star} \mathrm{GG} 2359$ & - & AY 661688.1 & AY 661688.1 & - \\
\hline & $\wedge$ Wedin 7651 (UPS) & - & - & - & НМ 244814.1 \\
\hline S. confusa & ^ Wedin 7070 (UPS) & - & DQ401143.1 & DQ401143.1 & HM 244815.1 \\
\hline \multirow[t]{4}{*}{ S. populorum } & $\wedge$ UME41471 & U86582.1 & - & - & - \\
\hline & ${ }^{*}$ MW7301 & - & AY 527334.1 & - & - \\
\hline & ^ Gilenstam 2353 (UPS) & - & - & $\mathrm{AY}_{3} 00833.1$ & - \\
\hline & ^Wedin 7626 (UPS) & - & - & - & HM 244817.1 \\
\hline \multirow[t]{3}{*}{ S. radiata } & $\wedge$ Palice (ESS 21520) & AY300864.1 & - & - & - \\
\hline & ${ }^{*}$ AFTOL 398 & - & DQ782846.1 & - & - \\
\hline & ^Jamie Platt JP222 & - & - & AF356663.1 & AY641079.1 \\
\hline \multirow[t]{3}{*}{ S. sp. } & ${ }^{\star} \mathrm{GG} 2445 \mathrm{a}$ & - & AY 527318.1 & AY 527318.1 & - \\
\hline & ${ }^{*} \mathrm{GG} 2440 \mathrm{~b}$ & - & AY 527321.1 & - & - \\
\hline & ${ }^{*} \mathrm{GG} 2620 \mathrm{~b}$ & - & AY 527332.1 & AY 527332.1 & - \\
\hline S. urceolatum & *AFTOL 96 & DQ983488.1 & HQ650601.1 & - & DQ992478.1 \\
\hline
\end{tabular}




\section{Taxonomy}
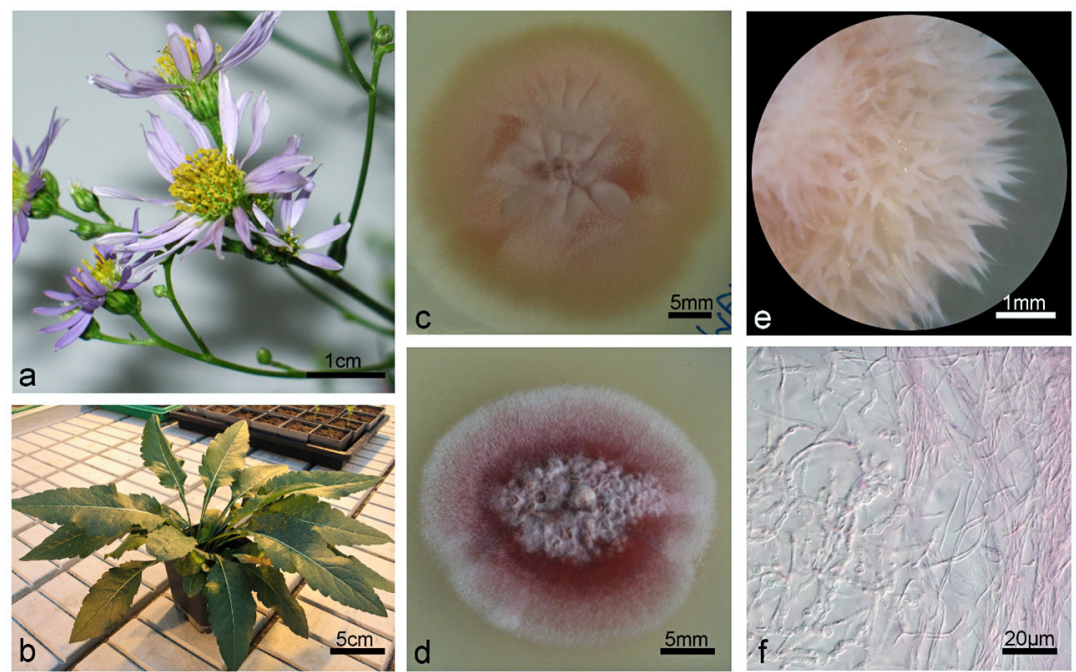

Fig. 2. The endophytic fungus Cyanodermella asteris. a, b. Habitus of the host Aster tataricus showing inflorescences and ground rosette; c. 55 d-old growth on malt extract agar; d. 55 d-old growth on potato dextrose agar; e. filamentous structure of the colony ( $36 \mathrm{~d}$ old $)$; f. hyphae stained with Congo Red.

Cyanodermella asteris L. Jahn \& Ludw.-Müll. sp. nov.

FIG. 2 MycoBAnK MB 814158

Differs from C. viridula by its endophytic habit and its rose-pink hyphae in culture.

TYPE-Germany, Saxony, Dresden, isolated as endophyte of Aster tataricus cv. Austria, 21 Mar 2013, L. Jahn, holotype Herb. DR 043292 (metabolically inactive), living culture at DSMZ under DSM 100826.

Етумодоgy - The epithet 'asteris' is derived from the host plant, Aster tataricus cv. Austria.

Sexual stage unknown. Colonies (14 d old) on potato dextrose agar white $(\mathrm{N} 9)$ to pale pink (2.5R 9/2); in age fading from pale pink to light pink (2.5R 8/6); after 30 days, becoming white towards the margin and turning light and deep pink (2.5R 6/10) to vivid red (5.0R 4/14) towards the center. Colors less intense on malt extract agar: 7-14 d old colonies usually colorless (N 9) and hardly distinguishable from the medium, $>50 \mathrm{~d}$ old colonies colorless at the margin and light pink at the center. 
$114 \ldots$... Jahn \& al.

Growth rate $0.7 \pm 0.3 \mathrm{~mm} /$ day on potato dextrose agar and $0.9 \pm 0.1 \mathrm{~mm} /$ day on malt extract agar at an optimum temperature of $22-24^{\circ} \mathrm{C}$ and a $\mathrm{pH}$ of $5.8-6$.

HOST \& DISTRIBUTION-The distribution of the endophytic fungus C. asteris may be linked to the distribution of its host, $A$. tataricus, which is native to northern Asia. Cyanodermella asteris has been isolated and detected by PCR in several plants of A. tataricus cv. Austria and also detected by PCR in A. tataricus plants obtained from the Botanical Garden Dresden, Germany, but which originated in Siberia. Isolation of $C$. asteris from these plants is currently in progress.

\section{Phylogenetic results}

The nuclear rDNA sequences of Cyanodermella asteris used for identification (as the "rDNA cluster") comprised the 18S (1539 bp), ITS (ca. 535 bp) and 28S (1359 bp) (GenBank KT758843). The first BLASTn search showed a distant relationship to the Stictidaceae lineage of the ostropalean fungi (TABLE 2). This led to a second BLASTn search against the Stictidaceae (TABLE 3). The 18S C. asteris sequence showed as nearest neighbor to C. oleoligni (98\% identity) and C. viridula (95\% identity), Acarosporina microspora (94\% identity), and less closely related Stictis species. Similarly, the $28 \mathrm{~S}$ C. asteris sequence revealed a relationship with C. oleoligni (94\% identity) and C. viridula (95\% identity). The ITS region showed a closest match to C. oleoligni (91\% identity), Stictis sp. (96\% identity), and Carestiella socia (96\% identity); no ITS sequences were available for C. viridula.

TABLE 2. BLASTn results of the C. asteris rDNA cluster region

\begin{tabular}{|c|c|c|c|c|c|c|c|}
\hline \multirow[b]{2}{*}{ LocI } & \multicolumn{2}{|c|}{ C. ASTERIS } & \multirow[b]{2}{*}{ Species } & \multicolumn{4}{|c|}{ BLASTN HITS } \\
\hline & \# BPs & GC $\%$ & & ACCESSION \# & $\begin{array}{l}\text { QUERY } \\
\text { COVER }\end{array}$ & IDENT & E VAlue \\
\hline \multirow[t]{5}{*}{$18 \mathrm{~S}$} & 1521 & 48.0 & Cyanodermella oleoligni & KX999145.1 & $74 \%$ & $98 \%$ & 0.0 \\
\hline & & & Trapelia involuta & AF119499.2 & $78 \%$ & $91 \%$ & 0.0 \\
\hline & & & Trapelia placodioides & AF119500.2 & $78 \%$ & $95 \%$ & 0.0 \\
\hline & & & Acarospora smaragdula & AY552543.1 & $78 \%$ & $91 \%$ & 0.0 \\
\hline & & & Cyanodermella viridula & U86583.1 & $78 \%$ & $91 \%$ & 0.0 \\
\hline \multirow[t]{3}{*}{ ITS } & 899 & 53.9 & Pleopsidium chlorophanum & DQ525472.1 & $47 \%$ & $84 \%$ & $4^{\star} 10^{-97}$ \\
\hline & & & Acarospora insignis & LN890273.1 & $39 \%$ & $87 \%$ & $6^{\star} 10^{-95}$ \\
\hline & & & Acarospora smaragdula & EU870652.1 & $38 \%$ & $86 \%$ & $4^{\star} 10^{-97}$ \\
\hline \multirow[t]{3}{*}{$28 \mathrm{~S}$} & 1329 & 49.4 & Cyanodermella oleoligni & KX950461.1 & $89 \%$ & $94 \%$ & 0.0 \\
\hline & & & Umbilicaria crustulosa & HM161593.1 & $100 \%$ & $90 \%$ & 0.0 \\
\hline & & & Umbilicaria haplocarpa & HM161534.1 & $99 \%$ & $90 \%$ & 0.0 \\
\hline
\end{tabular}


TABLE 3. BLASTn results of the C. asteris rDNA cluster region against Stictidaceae

\begin{tabular}{|c|c|c|c|c|c|}
\hline \multirow[b]{2}{*}{ LOCI } & \multirow[b]{2}{*}{ SPECIES } & \multicolumn{4}{|c|}{ BLASTN HITS } \\
\hline & & ACCESSION \# & $\begin{array}{l}\text { QUERY } \\
\text { COVER }\end{array}$ & IDENT & E VALUE \\
\hline \multirow{4}{*}{$18 \mathrm{~S}$} & Cyanodermella oleoligni & KX999145.1 & $74 \%$ & $98 \%$ & 0.0 \\
\hline & Cyanodermella viridula & U86583.1 & $77 \%$ & $95 \%$ & 0.0 \\
\hline & Acarosporina microspora & AY 584667.1 & $76 \%$ & $94 \%$ & 0.0 \\
\hline & Stictis urceolatum & DQ983488.1 & $76 \%$ & $94 \%$ & 0.0 \\
\hline \multirow{5}{*}{ ITS } & Cyanodermella oleoligni & KX950434.1 & $32 \%$ & $91 \%$ & $2^{\star} 10^{-95}$ \\
\hline & Stictis radiata & AY 527308.1 & $30 \%$ & $88 \%$ & $3^{\star} 10^{-88}$ \\
\hline & Stictis brunnescens & AY 661688.1 & $20 \%$ & $96 \%$ & $3^{*} 10^{-84}$ \\
\hline & Cryptodiscus pini & FJ904682.1 & $28 \%$ & $88 \%$ & $4^{*} 10^{-82}$ \\
\hline & Carestiella socia & AY 661682.1 & $20 \%$ & $96 \%$ & $4^{*} 10^{-82}$ \\
\hline \multirow{5}{*}{$28 \mathrm{~S}$} & Cyanodermella oleoligni & KX950461.1 & $89 \%$ & $94 \%$ & 0.0 \\
\hline & Xyloschistes platytropa & KJ766680.1 & $97 \%$ & $88 \%$ & 0.0 \\
\hline & Stictis radiata & AY340575.1 & $77 \%$ & $90 \%$ & 0.0 \\
\hline & Acarosporina microspore & AY 584643.1 & $83 \%$ & $90 \%$ & 0.0 \\
\hline & Cyanodermella viridula & HM244763.1 & $41 \%$ & $95 \%$ & 0.0 \\
\hline
\end{tabular}

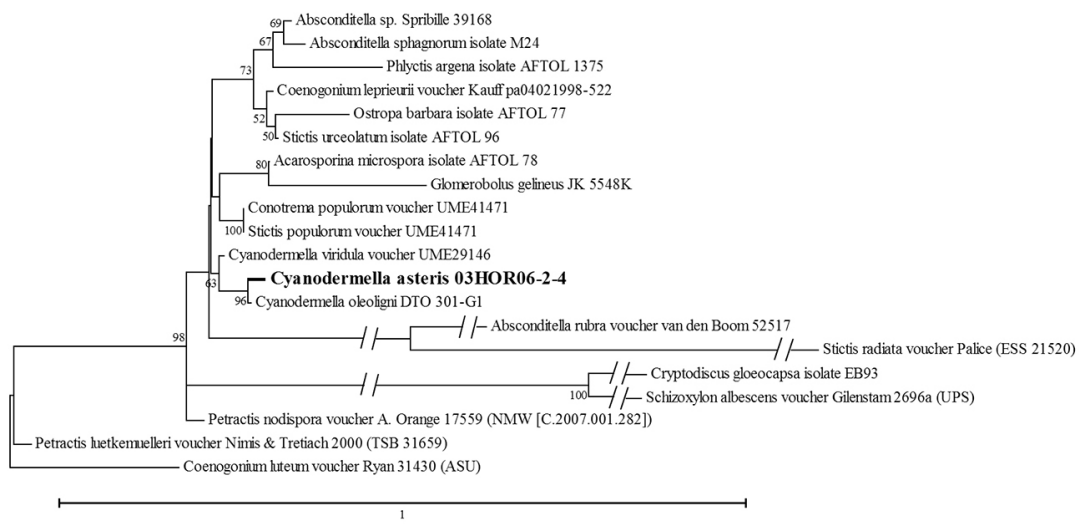

FIG. 3. 18S sequence-based phylogeny of Cyanodermella asteris and closely related taxa in the Stictidaceae using Maximum Composite Likelihood based on the Kimura-2-parameter model (Kimura 1980). The tree with the highest log likelihood (-15822.6420) is shown. A discrete Gamma distribution was used to model evolutionary rate differences among sites [5 categories $(+\mathrm{G}$, parameter $=0.6859)]$. The percentage of trees clustering the associated taxa is shown next to the branches. Coenogoniaceae and Phlyctidaceae were used as out-group. 
$116 \ldots$... Jahn \& al.

The RPB2 sequence (2059 bp, GenBank KU934214) was used to refine phylogenetic relationships within the Stictidaceae. Phylogenetic analyses based on the individual 18S, ITS, 28S, and RPB2 sequences of Cyanodermella asteris with other Stictidaceae strongly support a sister-relationship among C. asteris, C. viridula, and C. oleoligni (FIgs 3-6). The combined ML tree also

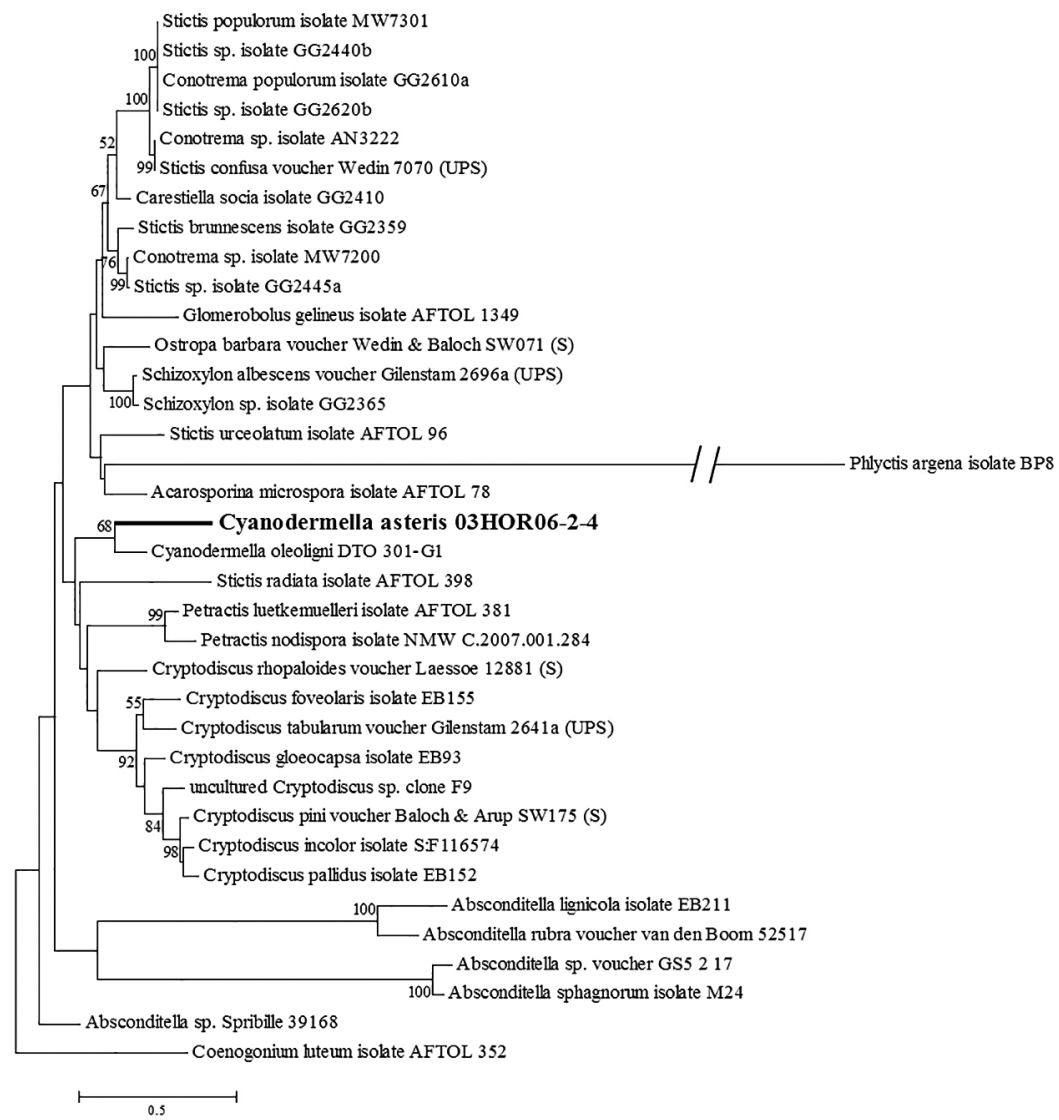

FIG. 4. ITS sequence-based phylogeny of Cyanodermella asteris and closely related taxa in the Stictidaceae using Maximum Composite Likelihood based on the General Time Reversible model (Nei \& Kumar 2000). The tree with the highest log likelihood (-11371.6910) is shown. A discrete Gamma distribution was used to model evolutionary rate differences among sites [5 categories $(+\mathrm{G}$, parameter $=1.4100)]$. The percentage of trees clustering the associated taxa is shown next to the branches. Coenogoniaceae were used as out-group. 
clusters C. asteris with the other two Cyanodermella species, but the combined phylogenetic tree is not as well supported as the others due to missing sequences (FIG. 7; Treebase TB2:S18310).

\section{Discussion}

Sequence analyses of the nuclear rDNA cluster from Cyanodermella asteris implied a close relationship with the Stictidaceae lineage of the Ostropales (Lecanoromycetes, Pezizomycotina, Ascomycota). The Stictidaceae are either saprotrophic or lichenized, and no stictidaceous species have been described as plant endophytes. However, Schulz \& Boyle (2005) noted that

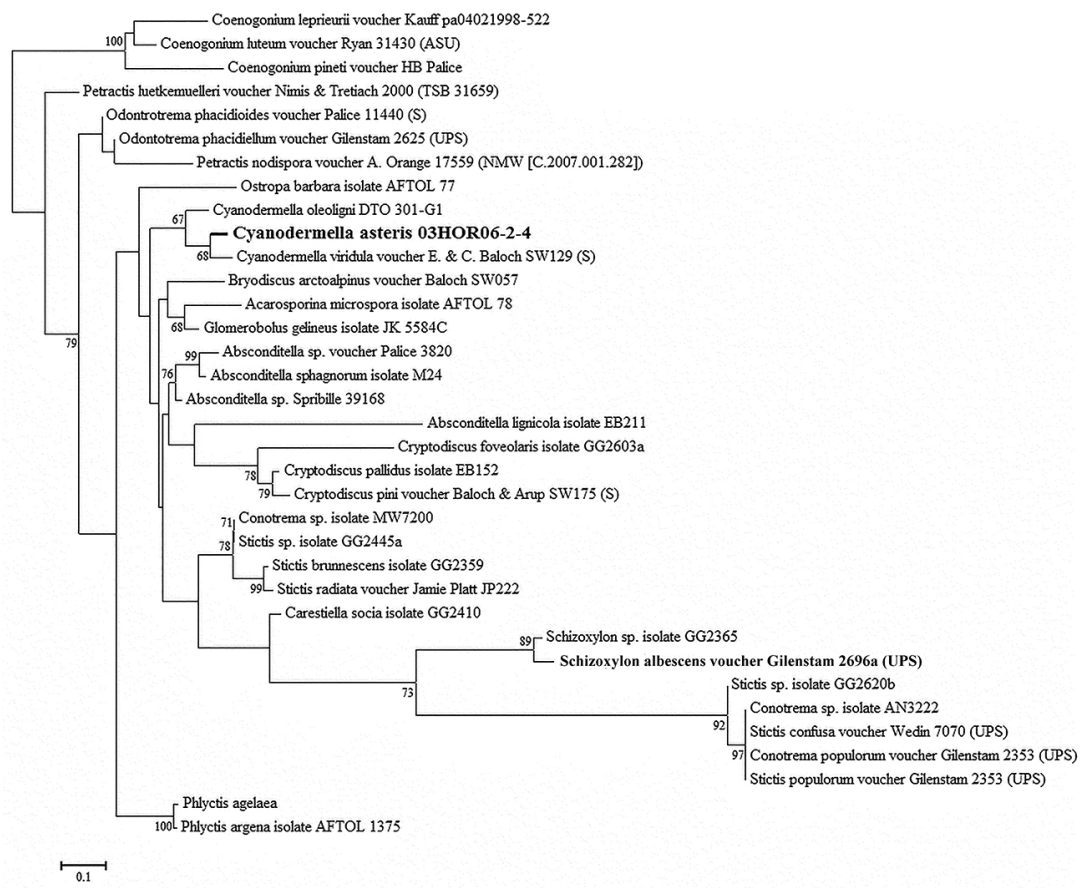

FIG. 5. 28S sequence-based phylogeny of Cyanodermella asteris and closely related taxa in the Stictidaceae using Maximum Composite Likelihood based on the General Time Reversible model (Nei \& Kumar 2000). The tree with the highest log likelihood (-15372.4003) is shown. A discrete Gamma distribution was used to model evolutionary rate differences among sites (5 categories $[+\mathrm{G}$, parameter $=0.6449)]$. The percentage of trees clustering the associated taxa is shown next to the branches. Coenogoniaceae and Phlyctidaceae were used as out-group. 


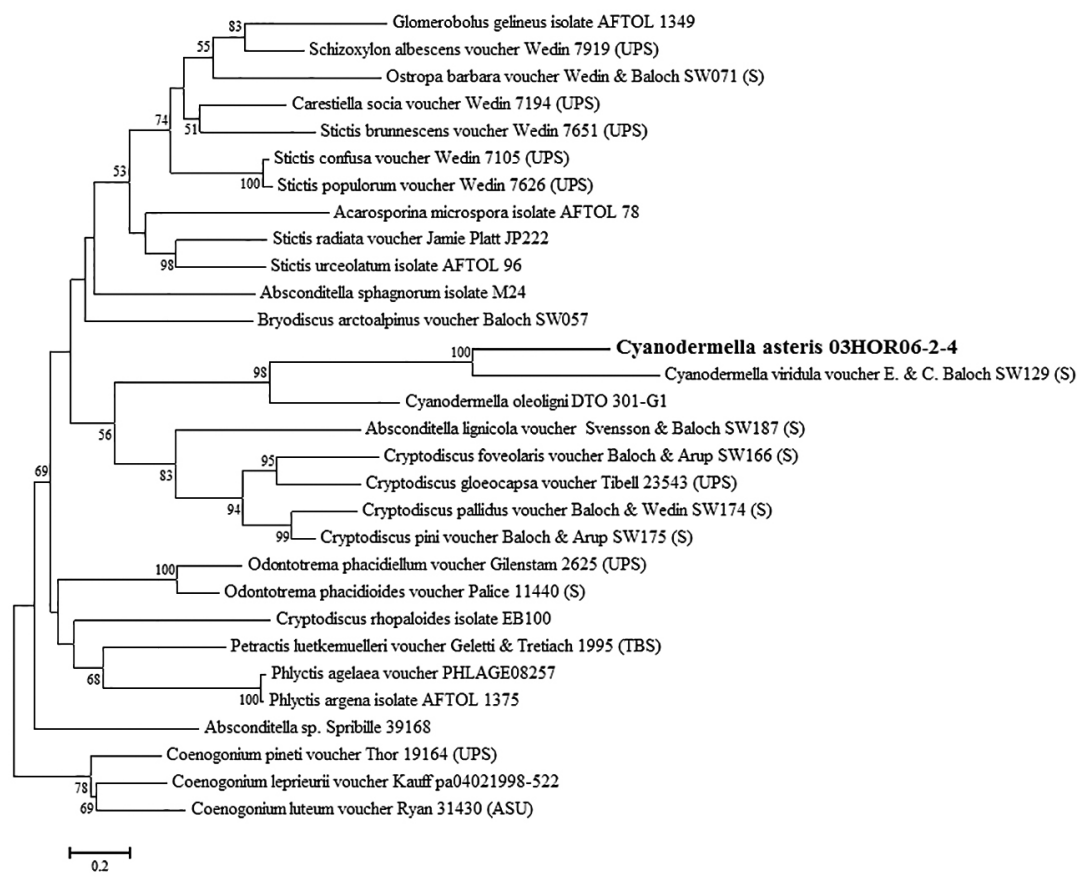

FIG. 6. RPB2 sequence-based phylogeny of Cyanodermella asteris and closely related taxa in the Stictidaceae using Maximum Composite Likelihood based on the General Time Reversible model (Nei \& Kumar 2000). The tree with the highest log likelihood (-29390.8544) is shown. A discrete Gamma distribution was used to model evolutionary rate differences among sites [5 categories $(+\mathrm{G}$, parameter $=0.5486)]$. The percentage of trees clustering the associated taxa together is shown next to the branches. Coenogoniaceae and Phlyctidaceae were used as outgroup.

many endophytes are facultative in that they adopt a saprotrophic strategy as soon as their host plant dies. Although C. asteris did not visibly develop any fruit bodies or other structures on dying and dead plant material of Aster tataricus, a saprotrophic habit cannot be excluded.

The species within the Stictidaceae live in different ecological niches over the whole world (see Sherwood 1977a, b, Eriksson 1967, 1981, Wedin et al. 2006, Czarnota, Kukwa 2008, Baloch et al. 2009; Fig. 8). Nonetheless, no Stictidaceae have been recorded from Siberia or Mongolia. Our species is 


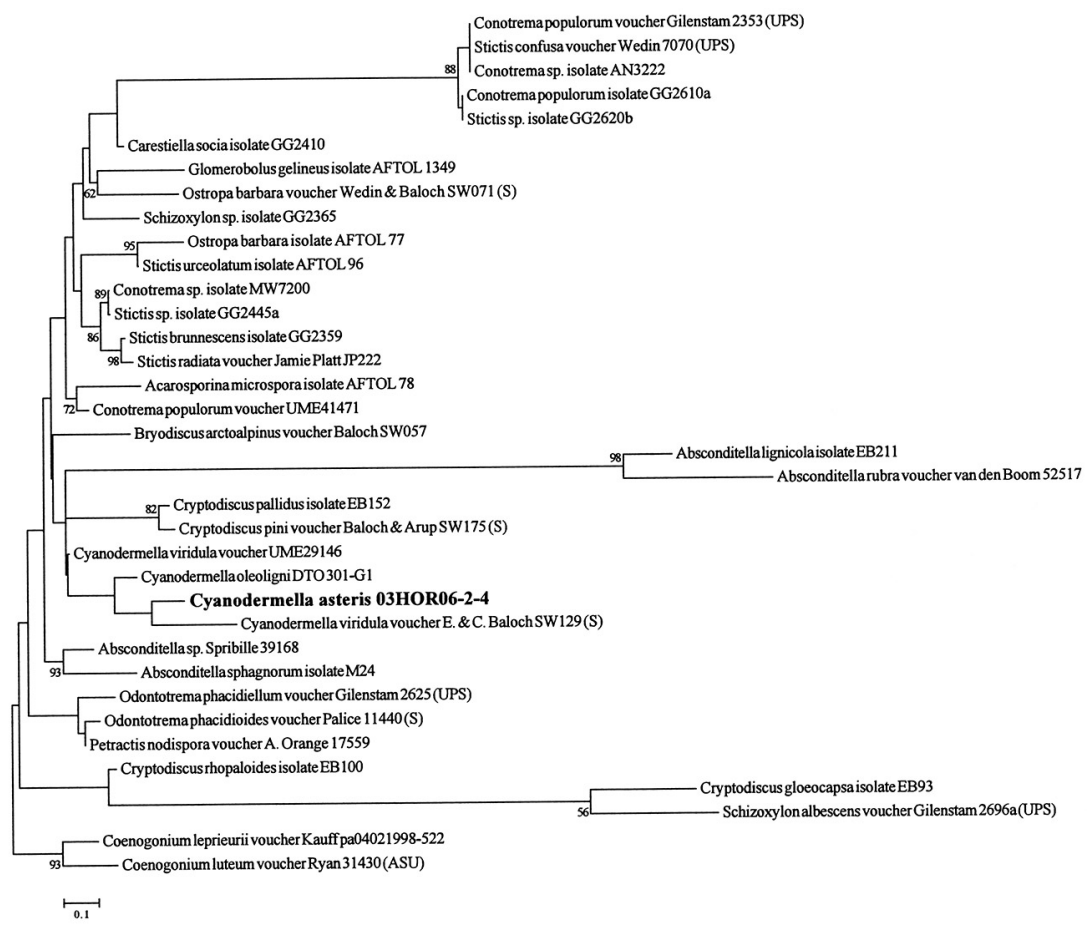

FIG. 7. Combined rDNA cluster + RPB2 sequence-based phylogeny of Cyanodermella asteris and closely related taxa in the Stictidaceae using Maximum Composite Likelihood based on the General Time Reversible model (Nei \& Kumar 2000). The tree with the highest log likelihood (-50824.7554) is shown. A discrete Gamma distribution was used to model evolutionary rate differences among sites $[5$ categories $(+\mathrm{G}$, parameter $=0.6150)]$. The percentage of trees clustering the associated taxa together is shown next to the branches. Coenogoniaceae were used as out-group.

both the first to inhabit a plant with origins in northern Asia and the first isolated endophyte representing the Stictidaceae.

It should be noted that within the Ostropales, the Stictidaceae are poorly sampled with relatively few sequences available to generate a phylogeny. The nearest neighbors of $C$. asteris were identified as C. viridula (Berk. \& M.A. Curtis) O.E. Erikss. and C. oleoligni van Nieuwenhuijzen \& Samson. The only sequences available for C. viridula - the $18 \mathrm{~S}$ and $28 \mathrm{~S}$ - show a $94 \%$ identity match with those from $C$. asteris. The more diverse ITS region-usually used 

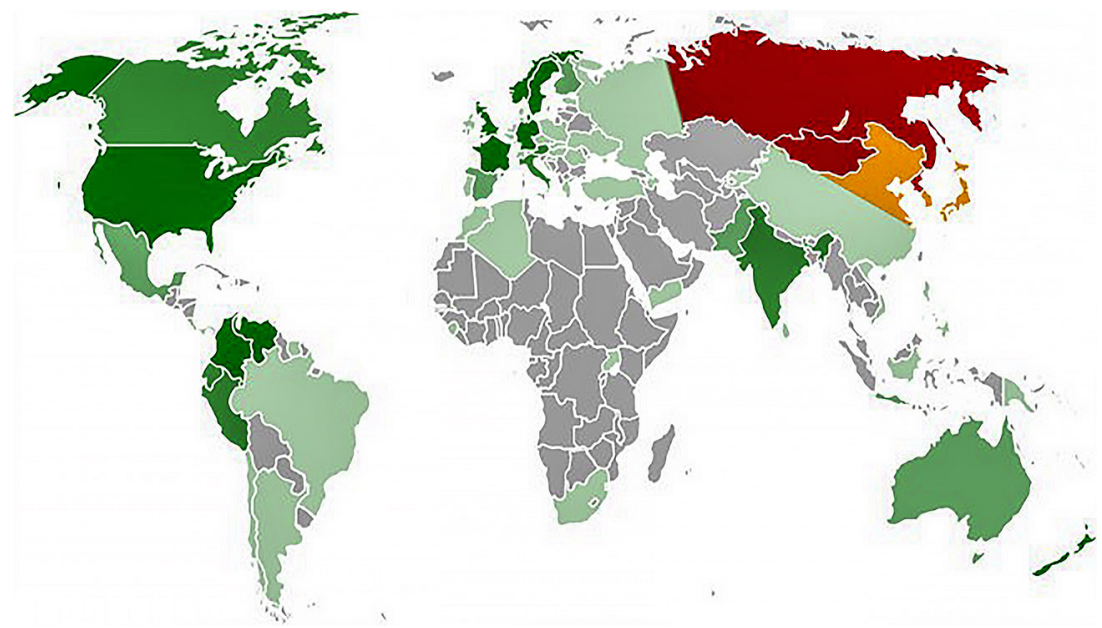

Fig. 8. Worldwide distribution of the Stictidaceae and Aster tataricus (Sherwood 1977a, b, Eriksson 1967, 1981, Wedin et al. 2006, Czarnota, Kukwa 2008, Baloch et al. 2009, Flann 2009). Aster tataricus is native to northern Asia including Siberia, Mongolia, Japan, Korea, and northern China, from which only a few Stictidaceae are described. Legend: Areas with $>15(\bullet)$, 10-14(•), 5-9 $(\bullet)$, or 1-4 $(\bullet)$ different species of Stictidaceae; native distribution of Aster tataricus $(\bullet)$; overlapping areas $(\bullet)$ and $(\bullet)$ are coded $(\bullet)$.

to identify and classify unknown fungal species (Schoch et al. 2012) - is not yet published from C. viridula. Cyanodermella oleoligni shows slightly higher similarities with C. asteris than C. viridula.

Cyanodermella is characterized by its perithecoid apothecia (Höhnel 1919; Eriksson 1967, 1981), which are very small and closed (Baloch et al. 2010). Only three species are described so far: C. oleoligni, C. viridula, and C. candida (Setch.) O.E. Erikss.

Cyanodermella viridula is known from old leaves of Leymus arenarius on beaches found in Sweden and western Russia near St. Petersburg as well as on twigs in North America, while C. candida is known only on ferns in Mexico (Eriksson 1967, 1981). Cyanodermella oleoligni (van Nieuwenhuijzen et al. 2016), which was isolated from oil-treated timber of Pinus sylvestris, grows slowly in grey to green colonies on fungal media. With the sexual stage of C. asteris not yet known despite an intensive search for ascomata on living and dead plant material, we expect its classification to be refined as more sequences from the Stictidaceae become available. 
The role of C. asteris in or for the host plant is not yet clear. Because no endophyte-free cultivars of $A$. tataricus are available to us, we are unable to determine whether the fungus aids the plant's growth in the environment. Schulz \& Boyle (2005) and Rodriguez et al. (2009) report that many endophytic fungi do not promote growth even when the plant is under stress. We speculate that if host plant serves as a habitat to protect $C$. asteris from the environment, the need for the fungus to adjust to the host plant might explain the initially slow growth (later somewhat accelerated; data not shown) of C. asteris in culture media. Future research will enable us to determine which metabolic and other features are characteristic for C. asteris.

\section{Acknowledgments}

This work was supported by the ERA-IB project MESIAB (EIB 10.004). KH van Pée, L Flor, J Ludwig-Müller and L Jahn were funded by the Sächsische Staatsministerium für Wissenschaft und Kunst (SMWK) and the Sächsische Aufbaubank (SAB). $\mathrm{W}$ Wohlleben and $\mathrm{T}$ Schafhauser were funded by the Bundesministerium für Bildung und Forschung (BMBF, grant 0315934). T Weber is supported by a grant of the Novo Nordisk Foundation. MME Huijbers and WJH van Berkel were supported by grants from the Netherlands Organisation for Scientific Research (NWO) and The Graduate School VLAG (Wageningen, The Netherlands). D Fewer and K Sivonen were funded by Finnish Funding Agency for Technology and Innovation (TEKES 40007/11). We gratefully acknowledge the revision of the phylogenetic trees by Dr. A Yurkov (DSMZ, Braunschweig, Germany) as well as Drs. Robert Lücking (Botanischer Garten und Botanisches Museum Berlin, Germany) and Peter Johnston (Landcare Research, New Zealand) for their expert assistance in revision.

\section{Literature cited}

Altschul SF, Gish W, Miller W, Myers EW, Lipman DJ. 1990. Basic local alignment search tool. Journal of Molecular Biology 215: 403-410. https://doi.org/10.1016/S0022-2836(05)80360-2

Baloch E, Gilenstam G, Wedin M. 2009. Phylogeny and classification of Cryptodiscus, with a taxonomic synopsis of the Swedish species. Fungal Diversity 38: 51-68.

Baloch E, Lücking R, Lumbsch H, Wedin M. 2010. Major clades and phylogenetic relationships between lichenized and non-lichenized lineages in Ostropales (Ascomycota: Lecanoromycetes). Taxon 14: 83-94.

Clémençon H. 2009. Methods for working with macrofungi: laboratory cultivation and preparation of larger fungi for light microscopy. Eiching, Germany: IHW-Verlag.

Czarnota P, Kukwa M. 2008. Contribution to the knowledge of some poorly known lichens in Poland. I. The genus Absconditella. Folia Cryptogamica Estonia 44: 1-7.

Eriksson OE. 1967. On graminicolous pyrenomycetes from Fennoscandia. 2. Phragmosporous and scolecosporous species. Arkiv für Botanik 6: 381-440.

Eriksson OE 1981. The families of bitunicate ascomycetes. Opera Botanica 60: 1-209. https://doi.org/10.1111/j.1756-1051.1981.tb01167.x 
Felsenstein, J. (1985). Confidence limits on phylogenies: An approach using the bootstrap. Evolution, 39: 783-791. https://doi.org/10.2307/2408678

Flann C (ed). 2009. Global Compositae checklist. http://compositae.landcareresearch.co.nz/ [Accessed: 27/06/2011].

Grube M, Hawksworth DL. 2007. Trouble with lichen: The re-evaluation and re-interpretation of thallus form and fruit body types in the molecular era. Mycological Research 111: 1116-1132. https://doi.org/10.1016/j.mycres.2007.04.008

Hibbett DS, Binder M, Bischoff JF, Blackwell M, Cannon PF, Eriksson OE, Huhndorf S, James T, Kirk PM, Lücking R, Lumbsch, HT, Lutzoni F, Matheny PB, McLaughlin DJ, Powell MJ, Redhead S, Schoch CL, Spatafora JW, Stalpers JA, Vilgalys R, Aime MC, Aptroot A, Bauer R, Begerow D, Benny GL, Castlebury LA, Crous PW, Dai Y-C, Gams W, Geiser, DM, Griffith GW, Gueidan C, Hawksworth DL, Hestmark G, Hosaka K, Humber RA, Hyde KD, Ironside JE, Kõljalg U, Kurtzman CP, Larsson K-H, Lichtwardt R, Longcore J, Miadlikowska J, Miller A, Moncalvo J-M, Mozley-Standridge S, Oberwinkler F, Parmasto E, Reeb V, Rogers JD, Roux C, Ryvarden L, Sampaio JP, Schüssler A, Sugiyama J, Thorn RG, Tibell L, Untereiner WA, Walker C, Wang Z, Weir A, Weiss M, White MM, Winka K, Yao Y-J, Zhang N. 2007. A higher-level phylogenetic classification of the fungi. Mycological Research 111: 509-547. https://doi.org/10.1016/j.mycres.2007.03.004

Höhnel F von. 1919. Fragmente zur Mykologie. XXIII Mitteilung, Nr. 1154 bis 1188 . Sitzungsberichte der Kaiserlichen Akademie der Wissenschaften Math.-naturw. Klasse Abt. I128: 535-625.

Kimura M. 1980. A simple method for estimating evolutionary rate of base substitutions through comparative studies of nucleotide sequences. Journal of Molecular Evolution 16: 111-120. https://doi.org/10.1007/BF01731581

Landan G, Graur D. 2007. Local reliability measures from sets of co-optimal multiple sequence alignments. Biocomputing 2008: 15-24. https://doi.org/10.1142/9789812776136_0003

Liu YJ, Whelen S, Hall BD. 1999. Phylogenetic relationships among ascomycetes: Evidence from ana RNA polymerase II subunit. Molecular Biology and Evolution 16: 1799-1808. https://doi.org/10.1093/oxfordjournals.molbev.a026092

Lutzoni F, Kauff F, Cox CJ, McLaughlin D, Celio G, Dentinger B, Padamsee M et al. 2004. Assembling the fungal tree of life: Progress, classification, and evolution of subcellular traits. American Journal of Botany 91: 1446-1480. https://doi.org/10.3732/ajb.91.10.1446

Morita H, Nagashima S, Uchiumi Y, Kuroki O, Takeya K, Itokawa H. 1996. Cyclic peptides from higher plants. XXVIII. Antitumor activity and hepatic microsomal biotransformation of cyclic pentapeptides, astins, from Aster tataricus. Chemical \& Pharmaceutical Bulletin, 44: 1026-1032. https://doi.org/10.1248/cpb.44.1026

Munsell AH. 1905. A color notation. Boston: G.H. Ellis.

Murashige T, Skoog F. 1962. A revised medium for rapid growth and bio assays with tobacco tissue cultures. Physiologia Plantarum 15: 473-497. https://doi.org/10.1111/j.1399-3054.1962.tb08052.x

Nei M, Kumar S. 2000. Molecular evolution and phylogenetics. Oxford University Press. New York. Penn O, Privman E, Ashkenazy H, Landan G, Graur D, Pupko T. 2010. GUIDANCE: A web server for assessing alignment confidence scores. Nucleic Acids Research, 38/SUPPL. 2. https://doi.org/10.1093/nar/gkq443

Rodriguez RJ, White JF, Arnold AE, Redman RS. 2009. Fungal endophytes: Diversity and functional roles. New Phytologist 182: 314-330. https://doi.org/10.1111/j.1469-8137.2009.02773.x

Schoch CL, Seifert KA, Huhndorf S, Robert V, Spouge JL, Levesque CA, Chen W. 2012. Nuclear ribosomal internal transcribed spacer (ITS) region as a universal DNA barcode marker for fungi. Proceedings of the National Academy of Sciences of the United States of America 109: 6241-6246. https://doi.org/10.1073/pnas.1117018109 
Schulz B, Boyle C. 2005. The endophytic continuum. Mycological Research 109: 661-686. https://doi.org/10.1017/s095375620500273x

Sela I, Ashkenazy H, Katoh K, Pupko T. 2015. GUIDANCE2: Accurate detection of unreliable alignment regions accounting for the uncertainty of multiple parameters. Nucleic Acids Research, 43: W7-W14. https://doi.org/10.1093/nar/gkv318

Shao Y, Ho CT, Chin CK, Poobrasert O, Yang SW, Cordell GA. 1997a. Asterlingulatosides C and D, cytotoxic triterpenoid saponins from Aster lingulatus. Journal of Natural Products, 60: 743-746. https://doi.org/10.1021/np970080t

Shao Y, Ho CT, Chin CK, Rosen RT, Hu B, Qin GW. 1997b. Triterpenoid saponins from Aster auriculatus. Phytochemistry 44: 337-340. https://doi.org/10.1055/s-2006-957365

Sherwood MA. 1977a. The ostropalean fungi II: Schizoxylon, with notes on Stictis, Acarosporina, Coccopeziza and Carestiella. Mycotaxon 6: 215-260.

Sherwood MA. 1977b. The ostropalean fungi. Mycotaxon 5: 1-277.

Shirota O, Morita H, Takeya K, Itokawa H, Iitaka Y. 1997. Cytotoxic triterpene from Aster tataricus. Natural Medicines 51: 170-172.

Tamura K, Stecher G, Peterson D, Filipski A, Kumar S. 2013. MEGA6: Molecular evolutionary genetics analysis version 6.0. MBE 30: 2725-2729. https://doi.org/10.1093/molbev/mst197

Vilgalys R, Hester M. 1990. Rapid genetic identification and mapping of enzymatically amplified ribosomal DNA from several Cryptococcus species. Journal of Bacteriology, 172: 4238-4246. https://doi.org/10.1128/jb.172.8.4238-4246.1990

van Nieuwenhuijzen E, Miadlikowska J, Houbraken J, Adan O, Lutzoni F \& Samson R. 2016. Wood staining fungi revealed taxonomic novelties in Pezizomycotina: New order Superstratomycetales and new species Cyanodermella oleoligni. Studies in Mycology 85: 107-124. https://doi.org/10.1016/j.simyco.2016.11.008

Wang GY, Wu T, Lin PC, Chou GX, Wang ZT. 2003. Phenolic compounds isolated from rhizoma of Aster tataricus. China Journal of Chinese Materia Medica 28: 946-948.

Wedin M, Döring H, Gilenstam G. 2004. Saprotrophy and lichenization as options for the same fungal species on different substrata: Environmental plasticity and fungal lifestyles in the Stictis-Conotrema complex. New Phytologist 164: 459-465. https://doi.org/10.1111/j.1469-8137.2004.01198.x

Wedin M, Döring H, Gilenstam G. 2006. Stictis s. lat. (Ostropales, Ascomycota) in northern Scandinavia, with a key and notes on morphological variation in relation to lifestyle. Mycological Research 110: 773-789. https://doi.org/10.1016/j.mycres.2006.04.010

White TJ, Bruns S, Lee S, Taylor J. 1990. Amplification and direct sequencing of fungal ribosomal RNA genes for phylogenetics. 315-322, in: Innis MA et al. (eds). PCR Protocols: a Guide to Methods and Applications. https://doi.org/10.1016/b978-0-12-372180-8.50042-1

Xu HM, Zeng GZ, Zhou WB, He WJ, Tan NH. 2013. Astins K-P, six new chlorinated cyclopentapeptides from Aster tataricus. Tetrahedron, 69: 7964-7969. https://doi.org/10.1016/j.tet.2013.07.006

Zhou WB, Zeng GZ, Xu HM, He WJ, Tan NH. 2013. Astataricusones A-D and astataricusol A, five new anti-HBV shionane-type triterpenes from Aster tataricus L. f. Molecules 18: 14585-14596. https://doi.org/10.3390/molecules181214585

Zhou WB, Zeng GZ, Xu HM, He WJ, Zhang YM, Tan NH. 2014. Astershionones A-F, six new anti-HBV shionane-type triterpenes from Aster tataricus. Fitoterapia, 93: 98-104. https://doi.org/10.1016/j.fitote.2013.12.021 